\title{
Difusión, pervivencia y obsolescencia del sistema lancasteriano de enseñanza en Chile: del desorden sistemático a la uniformidad en torno al sistema simultáneo, 1832-1860
}

\author{
Diffusion, survival, and obsolescence of the Lancastrian system \\ of education in Chile: from systematic disorder to uniformity \\ around the simultaneous system, 1832-1860
}

Andrés Baeza Ruz*

\begin{abstract}
RESUMEN
El artículo aborda la continuidad del sistema lancasteriano de en-

señanza en Chile a partir de 1832, año en el que comenzó su abandono en el Instituto Nacional. Se argumenta que su pervivencia se debió a la difusión que tuvo en la década previa y al impulso que se le dio desde la Escuela Normal de Preceptores, al validarlo como uno de los dos sistemas de enseñanza vigentes que todo preceptor debía conocer. Sin embargo, esto implicó abrir un espacio de crítica pedagógica hacia aquellas escuelas que implementaban este sistema alejado de su modelo original, definido previamente por Joseph Lancaster y Andrew Bell en Inglaterra. Dicha crítica fue elaborada por los visitadores de escuela, quienes, a partir de 1850, impulsaron la uniformidad de las escuelas en torno al sistema simultáneo. En ese escenario, el sistema mutuo o lancasteriano comenzó un gradual proceso de obsolescencia hasta ser abandonado definitivamente en la década de 1860 .
\end{abstract}

Palabras clave: sistema lancasteriano, sistema simultáneo, preceptores, visitadores de escuela.

\footnotetext{
* Chileno. Doctor en Historia, Universidad de Bristol, Reino Unido. Profesor asistente e investigador, Centro de Estudios Americanos, Facultad de Artes Liberales, Universidad Adolfo Ibáñez, Chile. E-mail: andrez.baeza.r@uai.cl La investigación para este artículo fue financiada por la Comisión Nacional de Investigación Científica y Tecnológica (CONICYT, hoy ANID), Programa FONDECYT Postdoctorado, Proyecto $N^{\circ} 3170966$ titulado "El sistema lancasteriano en Chile: adopción, adaptación y persistencia de un proyecto global en un contexto local: c. 1820-1860", desarrollado en el Instituto de Historia, Pontificia Universidad Católica de Chile.
} 


\section{SUMMARY}

This article reviews Chile's Monitorial or Lancasterian education system starting from 1832 when the Instituto Nacional discontinued its use. We argue that its survival was due to the diffusion that it had in the previous decade and the impulse given from the Escuela Normal de Preceptores when validating it as one of the two current education systems that every preceptor had to know. However, this implied opening a space of pedagogical criticism towards those schools that implemented this system far from its original model, previously defined by Joseph Lancaster and Andrew Bell in England. This criticism was elaborated by the school visitors, who, from 1850 onwards, promoted school uniformity around the simultaneous system. In this scenario, the mutual or Lancastrian system began a gradual obsolescence process until its permanent disuse in the 1860s.
Keywords: Lancasterian system, simultaneous system, preceptors, school visitors. 
El proceso de independencia hispanoamericano implicó cambiar el marco conceptual del lenguaje político. De la legitimidad monárquica, de derecho divino, se pasó una nueva legitimidad republicana sustentada en la soberanía popular. Los antiguos súbditos devinieron en ciudadanos, una nueva categoría política que debía ser construida y moldeada en función de los parámetros definidos por las elites que controlaron la dirección y rumbo de las nuevas repúblicas (Racine, 2020). Para construir al nuevo ciudadano, una herramienta fundamental fue la educación, por lo que los nuevos gobiernos se embarcaron en la labor de reconstruir sus antiguos sistemas educativos heredados desde el periodo colonial (Roldán Vera, 2005 y 2011).

Existió cierto consenso entre los nuevos gobiernos respecto de que la educación del nuevo ciudadano, bajo los parámetros coloniales, no era compatible con el proyecto modernizador que implicaba establecer nuevas repúblicas. Por lo tanto, una opción fue la de adoptar un nuevo sistema de enseñanza que permitiese construir los nuevos cimientos de las estructuras educativas necesarias para educar al ciudadano y fortalecer a estas nuevas entidades políticas (Racine, 2020). El sistema de enseñanza elegido fue el lancasteriano ${ }^{1}$, que debe su nombre a Joseph Lancaster, un cuáquero avecindado en Londres quien, en 1803, publicó las bases de un sistema de enseñanza que presentaba las siguientes características: i) prometía educar a cientos de niños a la vez, en un solo espacio y bajo la supervisión de un solo maestro; ii) el uso de niños mayores o "aventajados" en calidad de monitores, encargados de impartir la enseñanza a sus pares; iii) dentro del espacio co-

\footnotetext{
1 A lo largo de este artículo nos referiremos a los tres sistemas de enseñanza vigentes durante el siglo XIX. El sistema lancasteriano, mutuo o monitorial, el sistema simultáneo y el sistema mixto. El primero se basa en tres aspectos: "división de los pupilos en varias clases, la selección de uno o más estudiantes de la misma escuela para instruir a cada clase y, finalmente, el trabajo simultáneo de todas las clases en la misma sala, al mismo tiempo, haciendo un progreso gradual, independientemente del número de pupilos". El simultáneo, consistió en "enseñar a varios individuos como si fueran solo una persona. El profesor instruye a todos los estudiantes simultáneamente y todos responden al profesor simultáneamente. De vez en cuando, si es adecuado, el profesor puede hacer preguntas a algunos de ellos individualmente". El mixto, se define como "una combinación de los sistemas simultáneo y mutuo, tomando de cada uno los aspectos buenos y ventajosos y excluyendo lo más posible sus desventajas". Existía desde el siglo XVIII un cuarto sistema, el individual, consistente en la enseñanza de cada alumno por separado, pero para varios pedagogos representaba aquello que había que superar, por lo que ni siquiera era considerado un sistema, sino que poco más que un método de instrucción. Sobre esto, véase Caruso (2014: 19-23).
} 
mún, organizaba a los alumnos en grupos, dispuestos en filas paralelas, de acuerdo con su nivel de aprendizaje y al mando de un monitor; iv) la enseñanza de la lectoescritura se basaba en el método diseñado por el propio Lancaster, para el cual se usaban extractos de la Biblia conocidos como "lecciones escriturales", y v) la apertura de la escuelas y financiamiento de las mismas estaba a cargo de sociedades filantrópicas formadas por suscriptores voluntarios de cada localidad. ${ }^{2}$

Por otra parte, uno de los puntos que generó mayor adhesión hacia el sistema lancasteriano por parte de las nuevas autoridades fue su marcado énfasis en el "orden" (Roldán Vera, 2005: 655). La organización interna de esta escuela, en la que las rutinas y movimientos de los alumnos, así como la disposición espacial del salón, estaban estrictamente codificados (Hassard y Rowlison, 2002), se entendió como funcional a la necesidad de fundar un nuevo orden político republicano. Entre las imágenes utilizadas para describir a esta escuela en Hispanoamérica y Chile se utilizó la de un "regimiento" y la de una "nación o república en miniatura" (Henríquez, 1822). En el primer caso, se destacó sobre todo la férrea disciplina y noción de orden que imperaba en la escuela, acentuando también la sumisión por parte de los alumnos, quienes debían realizar todas sus labores en silencio. En el segundo caso, el de la referencia a la nación o república en miniatura, se hacía alusión a una escuela funcionando como una comunidad, con cada uno de sus miembros cumpliendo un rol predefinido y en que se distinguía claramente la autoridad del maestro, todo lo cual iba intrínsecamente ligado a la noción de orden. En ese sentido, uno de los rasgos que definían a la escuela "monitoriana", de acuerdo a uno de sus promotores en Chile, era el "orden y regularidad con que se hacen todas las cosas, y la obediencia que se exige de una á otra en la escuela que es en la realidad una nacion o republica en miniatura" (Henríquez, 1822: f.11). ${ }^{3}$

2 Existe abundante bibliografía sobre el sistema lancasteriano, mutuo o monitorial, y, en particular, sobre su difusión en Hispanoamérica tras el proceso de independencia (Amunátegui, 1895; Browning, 1921; Weingberg, 1994). En las últimas dos décadas ha existido una verdadera renovación de los estudios de la difusión de este sistema, coincidente con el auge de la historia global y de la internacionalización educativa. La síntesis de las características de este sistema ha sido elaborada a partir de algunos de los más relevantes (Caruso, 2005; Caruso y Roldán Vera, 2005; Landhal, 2019, Racine, 2020; Roldán Vera, 2005 y 2011; Sedra, 2011, Tschurenev, 2008).

3 Hemos publicado una transcripción y presentación de esta fuente en Baeza (2018). 
La adopción de este sistema en Hispanoamérica obedeció también a un rápido proceso de difusión e internacionalización llevado a cabo por la British and Foreign School Society (BFSS). Esta sociedad, de raigambre protestante, había nacido en 1814 para difundir las ideas lancasterianas dentro y fuera de los márgenes del Imperio británico. ${ }^{4} \mathrm{Ha}-$ cia la década de 1820 ya había alcanzado todos los continentes, razón por la cual se conoce como el primer sistema "global" de enseñanza (Caruso, 2005; Tschurenev, 2008; Twells, 2009). Esto se debió también a la propia vocación "universalista" de la BFSS que, en conjunto con su sociedad hermana, la British and Foreign Bible Society (BFBS), aspiraban a evangelizar a la humanidad mediante la difusión de biblias y la apertura de escuelas donde estas fuesen usadas como material de estudio (Racine, 2008). La independencia de América se abrió como una nueva y promisoria oportunidad, ante la expectativa de que las nuevas elites dirigentes liberales serían proclives a abrir la puerta de sus repúblicas a los protestantes. Lo cierto es que varios líderes del proceso independentista habían vivido en Londres y algunos de ellos, como el propio Simón Bolívar y, al parecer, Bernardo O'Higgins, se familiarizaron con el sistema durante su estadía. Por esto, sumado a la admiración por el modelo de organización política británico, no era de extrañar que adoptaran también su sistema educativo (Collier, 1967; Racine, 2010).

En Chile el sistema fue ensayado brevemente en el Instituto Nacional, en 1820, a partir de la lectura de uno de los manuales de Lancaster, pero rápidamente se abandonó debido a la complejidad de su implementación (Amunátegui, 1895). Esto motivó a las autoridades a contratar a un agente de la BFSS para que inaugurara una escuela modelo al alero del Instituto, emulando el rol de la escuela central fundada por Lancaster en Southwark, Londres. Tras varias gestiones en Londres y Buenos Aires, finalmente optaron por la contratación de James Thomson por un año, el que asumiría el cargo de director de Educación Pública al alero del Instituto Nacional. Por otro lado, el propio O'Higgins decretaría, el 21 de noviembre de 1821, la obligatoriedad de este sistema para todas las escuelas, constituyendo así un primer momento

4 Originalmente, había sido fundada en 1811 bajo el nombre de Royal Lancasterian Society, pero, tras la expulsión de Lancaster, cambió de nombre (Kaestle, 1973). 
uniformador en la historia de la educación chilena: todas las escuelas debían regirse por un único sistema. ${ }^{5}$

Para el caso chileno, una idea reiterada es que el sistema lancasteriano dependió en demasía de Thomson y que su partida a Perú, en 1822, marcó el inicio del fin de este sistema en Chile. Esto ha reforzado la interpretación de que la adopción de este sistema fue un intento más bien experimental por dotar de una organización al incipiente sistema educativo chileno, y que no tuvo mayor trascendencia (Amunátegui, 1895; Browning, 1921; Gutiérrez, 2011; Aedo Richmond, 2000). En cambio, en otras latitudes, como Perú (Espinoza, 2013), Colombia (Pita, 2017), Argentina (Silveira, 2013) o México (Tanck de Estrada, 1973), se reconoce que la adopción este método fue fundamental para sustentar la estructura organizativa de los nacientes sistemas educativos durante gran parte del siglo XIX. Por otro lado, si bien se admite que hubo intentos por implementarlo al alero del Instituto Nacional, con posterioridad a la partida de Thomson, se asume que Andrés Bello fue el "sepulturero" del mismo, al recomendar su abandono en la junta educativa del Instituto Nacional en 1832 (Amunátegui, 1895). Esto implica que, a diferencia de otros países hispanoamericanos, el sistema lancasteriano se habría abandonado tempranamente. En Ecuador, Perú, México y Argentina la implementación de este sistema duró hasta 1828, 1850, 1870 y 1873 respectivamente (Newland, 1991: 345). Solo Loreto Egaña (2001), Sol Serrano et al. (2014) y Rodrigo Mayorga (2017) han puesto algún matiz al reconocer que el sistema lancasteriano habría continuado en formas híbridas más allá de 1832.

En nuestro caso, suscribimos esta hipótesis, pero nos interesa demostrar que el sistema lancasteriano se mantuvo vigente como un sistema más entre varios hasta por lo menos 1860, año en que entró en vigencia la Ley de Instrucción Primaria, que eliminó la referencia a métodos o sistemas específicos que debían aprender los preceptores. Esto se debió en gran parte a la circulación de manuales y cuadros de lectura que facilitaron su adaptación local a contextos que no necesariamente eran los más idóneos para una implementación efectiva. No

5 Hemos abordado el proceso de adopción del sistema lancasteriano en la década de 1820 en Chile en otros trabajos, por lo cual no profundizaremos en este artículo los detalles de dicho proceso. Véase Baeza 2014, 2017 y 2020. 
obstante, como señalan Marcelo Caruso y Eugenia Roldán Vera (2005), el proceso de apropiación de este sistema tuvo una dimensión "cultural", en la cual los receptores adaptaron las fórmulas originales de acuerdo con sus circunstancias.

En este sentido, es importante considerar que en Chile el sistema continuó funcionando más allá de cualquier decisión oficial, debido a la incapacidad del Estado para llegar a las provincias y ejercer su poder público con eficacia (Lamperiere, 2017). Esta realidad fue intuitivamente identificada por Sarmiento, quien validó este sistema como uno más - junto con el sistema simultáneo- al fundar la Escuela Normal de Preceptores. De ahí en adelante la política estatal en torno a los sistemas de enseñanza pasó por varios momentos: el de asumir una realidad heterogénea, de coexistencia de diversos sistemas, para luego avanzar hacia una uniformidad paulatina, acotada a la vigencia de dos sistemas, hasta finalmente volver a uniformar a las escuelas en torno a un único sistema, el simultáneo. En este esquema, el sistema lancasteriano jugaría un papel fundamental, en la medida en que sería utilizado como instrumento transitorio hacia dicha uniformidad. Como señala Caruso (2015), este proceso de tránsito de un sistema de enseñanza a otro todavía no recibe suficiente atención de la historiografía y, en ese sentido, también buscamos contribuir en ello.

Para desarrollar el argumento, hemos organizado el artículo en cuatro secciones: la primera, analiza el proceso mediante el cual el sistema lancasteriano llegó a su supuesto fin de la mano de Andrés Bello, y se argumenta que esto obedeció a la decisión de impulsar el sistema simultáneo. Para ello se utilizan sobre todo fuentes secundarias acerca de este proceso. En segundo lugar, se abordan los elementos de continuidad del sistema lancasteriano hacia las décadas de 1830 y 1840 en las provincias, por medio de indicios y rastros encontrados en los archivos de los ministerios del Interior, de Justicia, Culto Educación Pública, y de las intendencias de Coquimbo, Concepción, Chiloé y Talca. La tercera parte explica el proceso de validación al interior de la Escuela Normal de Preceptores, a partir de un análisis del pensamiento de Sarmiento sobre los diversos sistemas educativos plasmado en obras de su autoría. Esto también se complementa con fuentes de archivo. Por último, argumentamos que fueron los visitadores de escuela los agentes fundamentales mediante los cuales el sistema lancasteriano 
comenzó una gradual pérdida de vigencia, hasta dar paso a una nueva uniformidad en torno al sistema simultáneo. Para esto utilizamos los informes de visitadores de escuela publicados en el Monitor de escuelas primarias y en los fondos documentales mencionados anteriormente.

\section{La "abolición" del sistema lancasteriano en el Instituto Nacional: de Ambrosio Lozier a Andrés Bello}

La estadía de James Thomson en Chile se extendió desde julio de 1821 a agosto de 1822, ya que, de acuerdo con su propio plan, deseaba viajar por toda Sudamérica y en dicha oportunidad recibió la invitación de San Martín para implementar el sistema en Perú (Thomson, 1827). Su ayudante, Antony Eaton correría una suerte similar, ya que llegó a Chile desde Inglaterra en septiembre de 1821 y abandonaría en junio de 1822, aunque por problemas de salud. La documentación disponible permite afirmar que la partida de Eaton y Thomson efectivamente significó un breve receso en la difusión del sistema lancasteriano, ya que hay un vacío en la información que se prolonga hasta 1826, año en que se nominó a Ambrosio Lozier, profesor y matemático francés, como rector interino del Instituto Nacional. Sin embargo, pese a esta pausa, fue esta administración la que dio un nuevo impulso a su implementación, en la medida que una de sus prioridades fue la de fomentar el uso de "métodos modernos" de enseñanza. De ahí en adelante, al menos hasta 1832, el sistema lancasteriano gozó de cierta continuidad en su implementación al interior del Instituto Nacional y de otras instituciones, como en su difusión. ${ }^{6}$

Lozier era un matemático francés, interesado en aplicar métodos innovadores que hasta ese entonces solo eran conocidos marginalmente en Hispanoamérica, como el del suizo Henrich Pestalozzi. Entre esos métodos también estaba el lancasteriano, para cuya implementación contrató a Francisco Solano Pérez como preceptor de la escuela primaria, con un sueldo de $\$ 300$ anuales. Solano sería el preceptor y director de esta escuela hasta su cierre en 1832. Además, se emplearía este mismo sistema en el primer año del curso de aritmética en el

6 Todo el proceso de adopción del sistema lancasteriano al interior del Instituto Nacional entre 1821 y 1833, así como el rol que jugó esta institución en su difusión ha sido abordado en Baeza 2015 y 2017. 
nivel "secundario". A pesar de los esfuerzos por modernizar el plan de estudios del Instituto, lo cierto es que la administración de Lozier finalizaría abruptamente, como consecuencia de una serie de problemas disciplinarios internos, lo que forzó su renuncia en septiembre del mismo año. De acuerdo con Amunátegui, Lozier habría viajado posteriormente a Concepción a "dirijir una escuela de enseñanza mutua por el sistema de Lancaster" (Amunátegui, 1895: 174) que habla de su predilección por este sistema y de lo relevante que fue para su administración.

La asunción de su reemplazante, Juan Francisco Meneses, prelado católico, exrealista y conservador, se dio unos pocos meses antes de que Francisco Antonio Pinto asumiera interinamente la Presidencia de la República (febrero de 1827), tras la renuncia de Ramón Freire. A diferencia del primero, Pinto era un reconocido "pipiolo", calificativo con el cual eran conocidos los liberales del periodo. Previamente había ejercido como intendente de Coquimbo, donde pudo interiorizarse del funcionamiento del Instituto literario fundado en La Serena en abril de 1821, a emulación del Instituto Nacional, y donde estudió en profundidad el estado de la educación en la provincia (Ossa, 2007: 104-105). Como apunta Juan Luis Ossa, esta preocupación por el estado de la educación pública fue plasmada en su programa de gobierno:

"La instruccion pública es el objeto predilecto de los ciudadanos del Gobierno; pero en tan escaso tiempo apenas ha podido subministrarle algunas cortas mejoras en sus diferentes ramos. Ha sido necesario buscar elementos de que carecíamos para poder desenvolver en toda su extensión un plan de educación primaria, capaz de elevarla del miserable estado en que yace en toda la República (Ossa, 2007: 119). ${ }^{7}$

Quizás por su experiencia como intendente, la preocupación de Pinto por la educación pública iba más allá del rol del Instituto Nacional, el que a esas alturas ya era considerado más bien un bastión del peluconismo (Serrano, 2016: 58). Sabiendo, además, que su hombre favorito en materias educacionales, José Joaquín de Mora, difícilmente sería admitido como profesor, optó por apoyar a este en su estrate-

$7 \quad$ El documento citado por Ossa es el "Mensaje del Ejecutivo al Congreso Constituyente", fechado el 25 de febrero de 1828. 
gia de fundar una institución secundaria alternativa, el Liceo de Chile (Roldán Vera, 2005; Baeza, 2017). Esto tuvo serias repercusiones al interior del Instituto, no tanto por la "competencia" que se había instalado, sino que por la opción de Pinto de financiar el proyecto de De Mora con 42 becas. Ciertamente, esto afectaba el financiamiento del Instituto, dado que el dinero ahora debía distribuirse en estas dos instituciones.

Por otro lado, Pinto buscó otras estrategias para contrapesar el poder del Instituto. Uno de ellos fue la formación de una "Comisión de Instrucción Primaria", a inicios de 1829, paralela a la Junta de Educación del Instituto, pero con funciones similares y superpuestas. Por supuesto, uno de los miembros de dicha Comisión fue el propio Mora, además de Francisco Ruiz-Tagle y un anciano Manuel de Salas (Amunátegui, 1889: 391). A esta Comisión —y no a la Junta-correspondió, entre otras funciones, analizar la solicitud de José León Cabezón, un profesor español que había llegado a Chile en enero de 1828 proveniente de Buenos Aires, de fundar un colegio en Santiago. En su solicitud, "había prometido que en la escuela de primeras letras [adosada al colegio] se adoptaría el sistema de Lancaster". De acuerdo con Amunátegui, el 3 de mayo de 1829 la Comisión dio un dictamen favorable a la petición "i aconsejaron al gobierno que le cediera dos salas de la Universidad de San Felipe, en una de las cuales se había establecido la escuela dirijida por Mr. Thomson, con los bancos, mesas i útiles que se conservaban de aquel tiempo" (Amunátegui, 1889: 391-392). Finalmente, el colegio de Cabezón se estableció en una casona ubicada en la esquina de la actual intersección de las calles Moneda y San Antonio.

Evidentemente, la fundación de este nuevo establecimiento significó una nueva afrenta para el Instituto, dado que nuevamente el gobierno optaba por utilizar los escasos recursos disponibles en una nueva institución, en lugar de fortalecerlo. Pero, por otro lado, refleja también el impulso que se buscó dar a la implementación del sistema lancasteriano hacia fines de la década. Cabezón había utilizado este sistema previamente en Buenos Aires y había grandes expectativas acerca de que lo hiciera en Santiago. No es coincidencia, por tanto, que se recomendara el antiguo salón ocupado por Thomson para su funcionamiento, pues la llegada de Cabezón se veía también como una manera de retomar de una vez por todas lo que había quedado aban- 
donado. En este sentido, no es menor que, hacia 1828, el salón que había servido de Escuela Normal en 1821-1822, mantuviera su mobiliario intacto a la espera de ser nuevamente utilizado. Además, tampoco es coincidencia el rol protagónico del propio Mora. Su liceo era una institución secundaria, pero completamente organizada bajo el modelo de Lancaster, el cual conocía a la perfección luego de su larga estadía en Londres durante la década de 1810 (Roldán Vera, 2005; Baeza, 2017). Fue allí donde trabajó como traductor de varios manuales, textos y catecismos en la librería de Rudolph Ackerman, que fueron utilizados en varias escuelas lancasterianas de Hispanoamérica (Roldán Vera, 2003; Iglesias Rogers, 2017: 330).

La Guerra Civil de 1829 trajo como consecuencia el ascenso al poder del peluconismo y, en el plano educacional, implicó el fin del apoyo estatal a proyectos alternativos al Instituto Nacional, como el de Mora. Esto se expresó en el retiro de las becas del Liceo de Chile, las cuales fueron reasignadas al Instituto y a los colegios provinciales (Amunátegui, 1889: 437-439). Con esto se reafirmó el poder de esta institución, tanto en su rol de colegio secundario, como de piedra angular del sistema. Las comisiones paralelas formadas por Pinto también cesaron en sus funciones y la Junta de Educación volvió a detentar el poder de antaño. En esta oportunidad, el actor clave de dicha junta sería Andrés Bello, quien había arribado a Chile en 1829 y que venía de ser el director del Colegio de Santiago, otro de los tantos proyectos paralelos al Instituto que se habían erigido previo a la Guerra civil (Jaksic, 2001: 130). Como rector asumiría Blas Reyes, quien durante los primeros años de su periodo volvió a implementar el sistema lancasteriano en una de las escuelas primarias sostenidas por el Instituto, aprovechando lo adelantado por Meneses en cuanto a la disponibilidad de espacio y mobiliario adecuado, así como de monitores bien capacitados. De acuerdo con Amunátegui, "la enseñanza de primeras letras por el método de Lancáster, que durante el rectorado de Meneses no había podido llevarse a la práctica, tuvo su completa realización durante el rectorado de don Blas Reyes" (Amunátegui, 1889: 555).

Sin embargo, fue precisamente en este periodo en que se adoptó la medida que, para el mismo Amunátegui, así como para Webster Browning (1921) y otros, significaría la muerte virtual de la implementación del sistema lancasteriano en Chile. A raíz de las dificultades económi- 
cas experimentadas por el Instituto, el 5 de julio de 1832 la Junta de Educación, liderada por Bello, decidió dividir en dos secciones la escuela lancasteriana adjunta al Instituto. En una primera instancia, el argumento se limitó a denunciar la mala organización de la escuela lancasteriana, pues la mezcla de niños diversos en una misma sala, "a mas de ser perjudicial a la esmerada educación que algunos padres tratan de dar a sus hijos, por su exorbitante número retarda los progresos que habrán de esperarse de un método más fácil i sencillo que el lancasteriano". De ese modo, la escuela se dividió en dos secciones: una escuela para treinta alumnos, organizada bajo "el método antiguo" (individual) y financiada por la suscripción de sus alumnos, y otra gratuita bajo el "método de Lancáster" y con un número de alumnos indefinido" (Amunátegui, 1889: 558-559).

Tras más de un año de funcionamiento bajo esta modalidad, finalmente la Junta decidió suprimir la escuela lancasteriana, aduciendo motivos económicos. Inicialmente el gobierno se opuso a la decisión; sin embargo, mediante un oficio dirigido al Ministerio del Interior, la Junta centró su argumento en el ámbito pedagógico, repitiendo casi al pie de la letra los argumentos que casi una década antes había elaborado Andrés Bello cuando se le solicitó un informe sobre el sistema lancasteriano mientras residía en Londres. El 11 de septiembre de 1820 había escrito a Antonio Irisarri que "los monitores no están ni pueden estar preparados para enseñar" (Vaughan, 1987: 148-149). En la misiva de la Junta al ministro del Interior, con fecha 23 de diciembre de 1833, se afirmó que:

"la junta ha visto el resultado práctico de este sistema de enseñanza, que léjos de corresponder a las esperanzas, no solo ha retardado el aprendizaje de los alumnos, sino que éste al fin ha sido defectuoso: consecuencia natural de un método que, abandonada la enseñanza de un niño al escaso saber de otro mayor, contrae vicios que no se le corrijen, i en la lectura, especialmente, sus progresos están restrinjidos por la corta capacidad del monitor" (Amunátegui, 1889: 561).

El foco de la crítica era, por tanto, el retraso en el aprendizaje de los alumnos, generado por la incapacidad de los monitores de llevar a cabo adecuadamente el proceso de enseñanza. En otras palabras, se criticó que gran parte del peso de la enseñanza recaía en los hombros 
de niños inexpertos, que además eran mal capacitados. Frente a esto, el gobierno aceptó la decisión.

La decisión de la Junta de Educación y del gobierno en 1833 implicó, por una parte, poner término a este sistema a partir de una rotunda crítica pedagógica, pero, por otra, era también la apuesta por un sistema hasta ese entonces escasamente difundido en Chile: el sistema simultáneo. Al establecer como número máximo el de setenta alumnos, "que es el máximum a que puede extenderse la inmediata inspección del maestro" (Amunátegui, 1889: 561), la apuesta era que un docente se hiciera cargo de un solo grupo al mismo tiempo y con la ayuda ocasional de alumnos más antiguos. Sin embargo, la decisión no especificaba si ese grupo de setenta alumnos al mando de un único profesor debía ser de la misma edad o de un mismo nivel de aprendizaje. Presumiblemente, se trataba de un grupo diverso, lo que implicaría que el profesor debería enfocarse en pequeños subgrupos según su nivel de aprendizaje dentro de una misma aula. La disposición en filas de la escuela lancasteriana podía ser una solución práctica en ese sentido, ya que cada una era en sí mismo un curso que agrupaba a niños de un mismo nivel de aprendizaje. Pero, por otro lado, era el propio docente - y no los monitores - el que debía enfocarse en cada grupo por separado, mientras el resto desarrollaba las actividades que este les dejaba.

En otras palabras, si bien había un claro interés por incorporar el sistema simultáneo en las aulas escolares chilenas, lo que en la práctica ocurrió fue abrir la puerta a la implementación de un sistema mixto, en que los principios de los tres sistemas de enseñanza vigentes en el mundo hasta ese momento se combinarían de tal modo de crear uno nuevo. Esta mixtura de sistemas se trasuntó a todo el territorio, como veremos en la próxima sección. Pero fue precisamente en el marco de dicha mixtura que el sistema lancasteriano logró perdurar en la práctica, a pesar de esta decisión gubernamental.

\section{La pervivencia del sistema lancasteriano: un "desorden sistemático"}

La historiografía ha consignado que la pervivencia del sistema mutuo o lancasteriano en Chile se llevó a cabo sobre todo en formas híbridas, o bien, que en paralelo a los sistemas mutuo y simultáneo se difundió un sistema mixto entre ambos que habría predominado en las escuelas 
(Egaña 2001; Serrano et al., 2014; Mayorga, 2017). Como señala Carlos Newland (1991: 345), "aunque criticado, el sistema se abandonaría lentamente en los lugares donde fue implantado, pues no había con qué reemplazarlo. Más que volver a los antiguos métodos, se lo siguió utilizando, muchas veces según la libre interpretación de los docentes". En el caso de Chile fue el propio Lozier, pese a sus esfuerzos por impulsarlo en el Instituto Nacional, el que advirtió que tal libertad de interpretación derivaría a la larga en un abandono de las ideas de Lancaster. El 25 de octubre de 1827 escribió a los intendentes de Coquimbo y Concepción buscando apoyo para instalar una escuela lancasteriana tras su salida del Instituto. En sus misivas afirmó que el sistema "no ha hecho ningún progreso en la América del sur, aunque continua siendo la admiración de las otras partes del mundo (...) si se examina el estado actual de estos establecimientos, apenas se halla alguna señal del genio de Lancaster, y se ve que la base; es decir, las lecciones graduadas no han sido redactadas ni aun para la lectura (Fondo de la Intendencia de Coquimbo, vol. 299 y Ministerio del Interior, vol. 45).

El análisis documental del periodo sugiere que el sistema mutuo perduró como un sistema más entre otros hasta por lo menos 1860. A pesar de los esfuerzos acometidos en el seno del Instituto Nacional por abandonar este sistema, la evidencia sugiere que este perduró en varios lugares y que entre el periodo 1830-1860 existió lo que se denominó un "desorden sistemático", que se intentó regular con la fundación de la Escuela Normal de Preceptores. Esto, según lo expresaba Manuel Montt en su calidad de ministro de Educación, se refería tanto a la coexistencia de diversos métodos y sistemas de enseñanza, incluyendo sus formas híbridas, como a la escasa comprensión de la "filosofía" de los mismos, y a la escasez de maestros idóneos: "mientras que el régimen de las escuelas sea un desorden sistemático, mientras que no haya filosofía en los métodos, ni los maestros sean otra cosa que hombres desengañados de la fortuna que buscan en esta ocupación un medio de subsistencia cuando se sienten sin aptitudes, ni arbitrios para ganarla en otra cualquiera, es imposible conseguir resultados satisfactorios" (Montt, 1841: 230).

La percepción de las autoridades era que, en gran medida, el problema que aquejaba a la educación era la falta de uniformidad en los métodos y sistemas educativos, y la poca preparación de los maestros 
para implementarlos. Esto explica que durante las décadas de $1830 \mathrm{y}$ 1840 se asumió que más que uniformar la enseñanza en torno a un solo método, como ya se había hecho en 1821, era necesario que en las escuelas se implementaran pocos sistemas y métodos, pero que estos fuesen implementados adecuadamente por los maestros. Esto derivó en la puesta en marcha de una uniformidad gradual en torno a los métodos más conocidos, lo que llevó a fomentar el estudio de los mismos, tanto en la Escuela Normal como en las localidades. El propio Joaquín Prieto, presidente de Chile desde 1831, señaló que "facilitado por buenos libros i métodos los procederes de la enseñanza (punto en que debo confesar que falta todavía mucho para contentar mis deseos), podemos prometernos resultados altamente favorables a la moral del pueblo..." (Prieto, 1833: 384). De ese modo, por un lado, se entendió que era necesario reforzar la supervisión de las escuelas, lo que llevó al propio Prieto a establecer una superintendencia "que dé un impulso regular y uniforme a la educación" (Prieto, 1834: 25). Por otro, se entendió también que era necesario proveer a las escuelas de los medios adecuados: "que se mejore en ellas la enseñanza, por medio de maestros idóneos, de libros elementales adecuados i de buenos métodos" (Prieto, 1836: 4-5). Todo este diagnóstico estará en la base de dos de las políticas educativas más importantes del periodo y que tuvieron directa relación con el desarrollo de los sistemas y métodos educativos: la fundación de la Escuela Normal de Preceptores, en 1842, y la creación de la figura del visitador de escuelas, en 1847, que analizaremos en las secciones siguientes.

En este contexto, reconstruir la historia de esta pervivencia no es asunto fácil, debido a que hasta 1852 la información es bastante dispersa y fragmentada. Solo a partir de esa fecha se generó un mecanismo de recopilación de información sistematizada, a partir de los informes de los visitadores de escuela. Por lo tanto, para las décadas de 1830 y 1840, lo que encontramos son pequeños fragmentos y huellas que dejan algún testimonio acerca del uso de este sistema en diferentes localidades. Dichos testimonios nos permiten comprender que el sistema mutuo o lancasteriano gozó de un alto grado de difusión y que en diversas localidades hubo verdaderos esfuerzos por implementarlo. Pero, del mismo modo, los indicios aportados por las fuentes permiten sostener que, efectivamente, la implementación del sistema era mediada en la mayoría de los casos por maestros inexpertos o con escaso 
conocimiento del mismo. A juicio de las autoridades, este resultaba ser uno de los principales problemas a superar. Lo anterior puede explicar un segundo punto y es que existen numerosos registros de comunicaciones sostenidas entre autoridades locales y centrales a partir de la década de 1840, que dan cuenta de que se impulsó una política de difusión desde el propio gobierno, tendiente sobre todo a facilitar algunos medios materiales para su implementación, como manuales y cuadros de lectura. Esto se explica por lo que abordaremos en el siguiente punto, respecto de la decisión de que la Escuela Normal de Preceptores fomentara el sistema mutuo y el simultáneo.

Este último punto es relevante, debido a que, hasta la fundación de dicha institución, se observa una disparidad en la recepción de la información proveniente de Santiago hacia las provincias. Esto se debe a que en la década de 1830 la capacidad infraestructural y burocrática del Estado todavía era muy limitada (Lamperiere, 2017; López, 2014) y su "llegada" a diversas localidades no era homogénea (Ponce de León, 2010). De este modo se explica por qué se continuó utilizando el sistema lancasteriano en varias de ellas, a pesar de la decisión tomada por las autoridades centrales en 1833, que virtualmente implicaban el abandono del sistema. Una clara muestra de lo anterior es la nota enviada al Intendente por la junta del Instituto de La Serena el 29 de enero de 1834. En dicha oportunidad, informaron de "la abolición del método de enseñanza mutua y reemplazo por el sistema individual que se practicaba antes en la escuela del Instituto Nacional" (Archivo del Liceo Gregorio Cordovez, vol. 2). Esto debido a que, con anterioridad, habían solicitado al ministro del Interior el envío de cuadros de lectura de Lancaster; sin embargo, la respuesta fue que no era conveniente debido a que el sistema ya se había "abolido". Esto indica, por un lado, que efectivamente para las autoridades se había tomado la decisión de abandonar el sistema lancasteriano y, por otro, que varios meses después de adoptada la decisión, en algunas provincias todavía se continuaba implementando debido a la falta de supervisión de las mismas autoridades.

En otras provincias, algunos testimonios también dan indicios de que el sistema continuó difundiéndose. En la provincia de Concepción la continuidad del sistema se explica por dos motivos principales: i) la fundación de una Sociedad de enseñanza mutua, el 19 de junio de 
1824, que emularía a la Sociedad lancasteriana fundada en Santiago, con el objetivo de difundir la enseñanza mutua en la provincia (Baeza, 2017: 346), y la llegada de Ambrosio Lozier tras su salida del Instituto Nacional. Aunque Amunátegui da indicios de la intencionalidad de este último de establecer una escuela lancasteriana, lo cierto es que mantuvo correspondencia con el intendente para abrirla, dotarla de materiales y financiarla. El 25 de septiembre de 1827 escribió al intendente de dicha ciudad:

"el gobierno ha aprobado el establecimiento de la enseñanza mutua en Concepción, pero que por la escasez de fondos en que se halla no ha podido darme los impresos indispensables, ni medios necesarios para obtenerlos. En esta circunstancia he recurrido a la Sociedad de alumnos para los métodos modernos, que me ha franqueado para este objeto cuantos fondos tiene en caja, pero no alcanzando aún, me veo en la procesión de suplicar a usted, se digne a abrir una suscripción voluntaria para coadyuvar a la impresión de todos los objetos indispensables para la enseñanza mutua de primeras letras, como doctrina cristiana, cuadros de aritmética, manual de aritmética, silabarios, cuadros de lectura, cuadros de gramática castellana, etc." (Fondo de la Intendencia de Concepción, vol. 110).

De esta comunicación llaman la atención dos aspectos. El primero, la mención a la Sociedad de Alumnos para los Métodos Modernos, sociedad que él mismo había establecido junto a un grupo de alumnos durante su rectorado en el Instituto Nacional, en 1826, de la cual no se tenía mayores noticias. La misiva de Lozier da a entender que continuaba en funciones, pero que, además, contaba con patrimonio propio para poder financiar parte de una escuela. El segundo aspecto dice relación con la apelación a la "suscripción voluntaria" de los vecinos de Concepción, mecanismo de financiamiento que había difundido Joseph Lancaster para difundir su sistema de enseñanza (Baeza, 2020). De acuerdo al mismo Lozier, esta suscripción pareció haberse concretado, pues aludió a ella en una segunda comunicación al intendente, con fecha 24 de abril de 1834; es decir, varios años después de su solicitud. En dicha carta señaló que "cuando recibí la suscripción de los habitantes de Concepción para impresos destinados a la enseñanza mutua, el señor Intendente me dio luego 
la más amplia facultad para emplearla" (Fondo de la Intendencia de Concepción, vol. 116). Respecto del funcionamiento de esta escuela no hay mayores rastros, salvo que, aparentemente, Lozier no perseveró en ella y se habría instalado en la Araucanía (Amunátegui, 1895: 174). Una de sus cartas al intendente, escrita en abril de 1833, tuvo como objeto la entrega del material que había adquirido gracias a la suscripción: "un cajón de impresos, restos de lo que quedaba en mi poder concernientes a la enseñanza mutua" (Fondo de la Intendencia de Concepción, vol. 107).

La circulación de materiales concernientes a este sistema fue más extensa que lo mostrado en el caso de Lozier y varias escuelas mantuvieron existencias de los cuadros de lectura en sus inventarios. Este fue el caso, por ejemplo, del preceptor de la Escuela de Hualqui, José María Gavilán, quien reportó al intendente, el 1 de marzo de 1838, que el inventario de la escuela tenía como existencia "ochenta cuadros de enseñanza mutual fijados a la pared de dicho local" (Fondo de la Intendencia de Concepción, vol. 160). Es decir, no se trataba de cuadros guardados en una bodega o biblioteca, sino de cuadros que se usaban frecuentemente al estar fijados en la pared de la sala, según rezaba el sistema lancasteriano. Posteriormente, el 29 de mayo 1843, sería la junta educativa del propio Instituto literario la que solicitaría dicho material para su biblioteca, con el argumento de que "los objetos de enseñanza mutua siguientes contienen la mayor parte de lo que se ha publicado para este asunto, sea para enseñar, sea para leer" (Fondo del Ministerio de Justicia, Culto e Instrucción Pública, vol. 17). Y ya hacia fines de la década de 1840, el 12 de agosto de 1849, se sugeriría abiertamente la "conveniencia para plantear este método en las escuelas" (Fondo de la Intendencia Concepción, vol. 274: 26). No es coincidencia, entonces, que el 24 de febrero de 1852 José Benito de Vergara, visitador de escuelas, informara que la escuela de Los Ángeles se denominaba "escuela de enseñanza mutua" y que llevaba varios años de funcionamiento (Fondo de la Intendencia de Concepción, vol. 394).

Otros testimonios más fragmentarios dan cuenta de que algunos elementos asociados al sistema lancasteriano se continuaron implementando, aunque de manera algo inconsistente. Por ejemplo, la figura del "ayudante, a veces asimilada a la del "monitor", aparece fre- 
cuentemente en las fuentes del periodo. Este fue el caso de la Escuela de primeras letras, en Talca. El 23 de octubre de 1837, el intendente de la provincia escribió al ministro de Justicia, Culto e Instrucción Pública, para informar sobre el nombramiento del párroco de la ciudad como director de la escuela de dicha escuela. Para que pudiera mantener ambas funciones, se propuso nombrar "un monitor o maestro que bajo la inspección del capellán desempeñase la enseñanza de leer, escribir i primeras reglas de aritmética" (Fondo del Ministerio de Justicia, Culto e Instrucción Pública, vol. 4). En este caso, vemos que se trata más bien de un ayudante que trabajase en conjunto con el capellán, y no de la figura del "niño enseñante", propia del sistema lancasteriano. Como veremos más adelante, la figura del ayudante también comenzó a ser asociada al sistema simultáneo, toda vez que en aquellas escuelas donde los alumnos eran distribuidos en grupos homogéneos, el preceptor requería de un ayudante que mantuviera la vigilancia de los grupos que quedaban desatendidos mientras este impartía la lección a uno de ellos. Asimismo, el 28 de febrero de 1851 se aludió a que los preceptores de las escuelas de Ancud no habían sido alumnos de la Escuela Normal, sino que "tienen muy regular instrucción y método por haber practicado en las escuelas de Ancud en clase de monitores o ayudantes" (Fondo de la Intendencia de Chiloé, vol. 27: f.35). Esto habla de lo difundido que se encontraba el sistema lancasteriano en Chiloé hacia la década de 1850, pero también de cómo, gradualmente, comenzaron a combinarse elementos de diferentes sistemas de enseñanza, lo que a la larga derivaría en la creación de sistemas híbridos o mixtos.

A partir de 1842 los rastros asociados a la continuidad del sistema lancasteriano se relacionarán directamente con la fundación y proyección de la Escuela Normal de Preceptores en la mayoría de los casos. Como veremos a continuación, el establecimiento de esta institución significó un primer paso dado desde el Estado, con el objetivo de uniformar los sistemas de enseñanza y de mejorar el proceso de enseñanza-aprendizaje. El sistema de enseñanza mutua, como era frecuentemente llamado por Sarmiento, fue uno de los sistemas oficiales que se enseñaría a los futuros preceptores, lo que significó, por un lado, un reconocimiento a su vigencia, pero, por otro, el primer paso de una transición hacia un único sistema, que implicaría gradualmente su abandono. 


\section{La Escuela Normal de Preceptores y la vigencia del sistema Mutuo: la transición hacia una nueva uniformidad}

La historiografía reconoce en Sarmiento a otro de los "enemigos" del sistema lancasteriano y responsable, al igual que Bello, del definitivo abandono de su uso en las escuelas chilenas (Amunátegui, 1895: 204205). Lo cierto es que, si bien Sarmiento compartía la mirada crítica de Bello respecto de los aspectos netamente pedagógicos del sistema, su visión era que había posibilitado la rápida expansión de la instrucción primaria en aquellos lugares donde se había implementado con éxito, como en Gran Bretaña y Estados Unidos. Así, a diferencia de Andrés Bello, que consideraba que las deficiencias pedagógicas del sistema lancasteriano obedecían a su organización innata, Sarmiento reconocía que el gran aporte de este sistema había sido la organización que ofrecía a las escuelas. En ese sentido, no descartaba de plano su uso y en eso mostró una mirada bastante más pragmática que la de Bello. Esto se explica en parte por el hecho de que, como afirma Pablo Toro, "en el proyecto educacional de Sarmiento habitaba una lectura negativa del legado colonial junto con claras convicciones respecto a la necesidad de una instrucción popular masiva" (Toro, 2018: 111).

Prueba de lo anterior fue su primera experiencia pedagógica en Chile, cuando asumió como preceptor de la escuela primaria de Santa Rosa de los Andes (Aconcagua) en 1832, la que era administrada y financiada por el municipio. Cuando asumió su cargo, la escuela funcionaba bajo el sistema lancasteriano, por lo que entendió que ese era el sistema con el cual los chilenos se encontraban más familiarizados. De acuerdo con su propio relato, incluido en su obra De la educación popular, publicada en 1849, "en Aconcagua hice yo, en 1832, un ensayo con la escasez de materiales de que era posible disponer. Había para el efecto, el gobierno de Chile, mandado imprimir una colección de cuadros de lectura, una de aritmética y otra de escritura para servir de tema al dictado". De este modo constató que, hacia 1832, el propio gobierno aún buscaba difundir este sistema. No obstante, reglón seguido, afirmó que "estos establecimientos han desaparecido, sin dejar rastro alguno y por lo incompleto de sus medios, ha nacido la duda acaso sobre la eficiencia del sistema" (Sarmiento, 2009: 224). En otro escrito manifestaría su decepción con los resultados de su experiencia en Los 
Andes luego de utilizar el método de enseñanza mutua para la lectura: "no obstante mi entusiasmo por la enseñanza mutua y los métodos afectos por entonces a ella, pude observar que los resultados no correspondían a mis esperanzas, menos por el sistema mismo que por lo imperfecto de los métodos usados" (Sarmiento, 1899: 39-40). En otras palabras, no tenía una preconcepción negativa acerca del sistema, sino que constató mediante la práctica sus deficiencias.

Pese a esta negativa percepción, sus viajes a Europa y Estados Unidos, entre 1845 y 1847 -que serían el principal insumo para escribir De la educación popular-, le permitieron observar en terreno el grado de extensión de diversos sistemas pedagógicos, incluido el lancasteriano. De ese modo, atribuyó a la adecuada implementación de este sistema la buena organización de las escuelas:

"El sistema de enseñanza mutua de Lancaster, ha dejado por lo menos un progreso para la organización interna de las escuelas, que hoy está aplicado generalmente a todo sistema de enseñanza, tal es la colocación de las bancas en columna cerrada en el centro de la escuela, con espacio suficiente en torno de las murallas para facilitar el pasaje y los movimientos, y con una plataforma elevada hacia el lado a que están vueltas las caras de todos los alumnos, donde se coloca la pizarra para las demostraciones generales, y la mesa y asiento del maestro" (Sarmiento, 2009: 213).

Por lo tanto, reconoció que la capacidad organizativa del sistema lancasteriano podía ser de utilidad, debido especialmente a la disposición de las bancas y el orden al interior de las aulas. Posteriormente, en 1854, en su calidad de editor de El monitor de escuelas primarias, también rescató aspectos a su juicio valorables de este sistema: el rol de las sociedades particulares en su difusión, lo que era compatible con un Estado docente cuya prioridad debía ser sobre todo la de brindar educación a los más pobres. El modelo filantrópico de estas sociedades podía ser clave a la hora de expandir la instrucción primaria, en la medida que coadyuvaran al Estado en su rol docente (Baeza, 2020).

Pero, más allá de estos aspectos, lo cierto es que su crítica pedagógica era rotunda. Y ello se hizo más evidente al comparar los diferentes sistemas educativos vigentes en ese entonces. Del sistema mutuo o lancasteriano afirmó que: "muy divididos están los pareceres en cuan- 
to a su eficacia como medio de enseñanza" y "por regla general, puede decirse que sólo es aplicable con provecho a las grandes masas de niños, de doscientos para adelante". El principal problema era, a su juicio, que se requerían demasiados elementos para que funcionara adecuadamente: "una disciplina rígida, un buen plantel de monitores, un material completo, en el que deben estar incluidos, en cuadros o tableros, todos los ramos de enseñanza, salas anchas y espaciosas". En ese sentido, sentenció, es necesario "que no falte ninguna de las piezas que componen el aparato para que el mecanismo de las operaciones juegue sin tropiezo" (Sarmiento, 2009: 223). Ahora bien, para Sarmiento el problema no era tanto la necesidad de disponer de estos elementos, como el método empleado para la enseñanza de la lectura y escritura. Fue en este ámbito en el que su crítica fue más lapidaria, al punto de hacer un llamado al gobierno a dejar de imprimir los cuadros de lectura del sistema mutuo que se continuaba repartiendo en las escuelas. En su visión, "el método de enseñanza mutua peca por una difusión empalagosa, suficiente para desalentar aun a los más interesados en aprender a leer" (Sarmiento, 1899: 45).

En contraste, el sistema simultáneo era, a su juicio, "el más antiguo, menos mecánico, más aplicable en toda circunstancia y generalmente más seguido" (Sarmiento, 2009: 224). Es decir, era de más fácil implementación que el mutuo, aunque también advirtió sobre algunas complejidades que, mal llevadas, podían hacerlo fracasar. En Alemania observó que cada grupo de alumnos era separado en salas distintas y que el maestro necesitaba varios ayudantes que se encargaran de los grupos que quedaban desatendidos mientras este enseñaba en una de las salas. En Holanda, en cambio, todo se llevaba a cabo en lo que él llamaba "escuelas monstruo"; es decir, un solo espacio, similar a la escuela lancasteriana, en que se enseñaba a cientos o miles a la vez. La diferencia era que, dentro de ese espacio, los alumnos eran separados en grupos que se desplegaban por todo el salón de acuerdo con su nivel de aprendizaje. No obstante, tenía la misma dificultad que el sistema lancasteriano: la necesidad de varios ayudantes o, bien, de varios maestros, para no dejar a ningún alumno sin atención. Pese a ello, como observó para Alemania, si la escuela lograba estar dotada de varios maestros idóneos atendiendo cada sala, se alcanzaba una situación ideal. Esto era, en la práctica, una idea incipiente de la escuela en su versión contemporánea: una 
escuela organizada en aulas, con grupos homogéneos y cada uno con un maestro a cargo. Sin embargo, en el periodo que analizamos todavía no se llegaba a este modelo, debido a que "tampoco había entonces ninguna conexión directa entre la instrucción simultánea y el uso de aulas. Como se ajusta a la instrucción grandes grupos, la instrucción simultánea se llevaba a cabo en aulas de escuela, no aulas de clase" (Hamilton, 1991: 30). De ese modo, cada maestro podía concentrarse en un grupo específico de alumnos y hacerse responsable de su aprendizaje, algo que no ofrecía el sistema lancasteriano al delegar la enseñanza en los monitores:

"Esta riqueza de dotación de maestros idóneos, da a aquellas escuelas la merecida reputación de que gozan. Un maestro se contrae a una clase que forma, por decirlo así, escuela separada y se constituye responsable de los procesos de aquellos alumnos que le están confiados. La influencia de la palabra y de la demostración de la pizarra ejercen todo su poder sobre una clase aislada sin perturbación exterior, sin desigualdades de instrucción" (Sarmiento, 2009: 224).

Aunque resaltó especialmente el caso alemán, la máxima realización de este ideal había observado dos décadas antes en la Escuela de la Patria de la provincia de San Juan, Argentina. Esta escuela había sido fundada en 1816 y el propio Sarmiento había sido su alumno. Una de sus particularidades fue que implementó desde un comienzo el sistema simultáneo, dividiendo a los alumnos en tres salones de acuerdo con el progreso de su aprendizaje, aunque con algunas modificaciones y elementos del sistema mutuo que lo hacían, en la práctica, un sistema mixto. No obstante, cuando el gobierno de Rivadavia impulsó la adopción del sistema lancasteriano en todas las escuelas, se inició una pugna, ya que los maestros se opusieron férreamente a cambiar de sistema. A ojos de Sarmiento, "hoy, que las imperfecciones de aquel sistema son mejor conocidas y contestadas sus ventajas, la razón queda de parte de los maestros. Su método de enseñanza era excelente y los resultados de muchos años les daban sanción y autoridad" (Sarmiento, 2009: 238). Finalmente, Rivadavia impuso su criterio, lo que Sarmiento atribuyó a argumentos políticos, dado que el impulso del sistema lancasteriano era parte de un programa liberal más amplio y la escuela ofrecía una educación eminentemente cató- 
lica. La consecuencia fue el abandono por parte de los maestros y el posterior cierre de la escuela.

Lo anterior cobra particular relevancia al considerar el rol que jugó Sarmiento en la fundación y dirección de la Escuela Normal de Preceptores en Chile, en 1842. La fundación de esta institución se inscribe en un proceso más amplio, de gradual consolidación del "Estado docente", mediante el cual el Estado de Chile comenzó a adquirir un mayor protagonismo, tanto en la conducción como en la provisión de enseñanza en todos sus niveles (Serrano et al., 2014). Ya en 1837 se había establecido el Ministerio de Instrucción Pública y en 1842 se había fundado la Universidad de Chile, que se constituiría en la nueva piedra angular del sistema educativo. Además, surgió la categoría de "escuela fiscal" como escuela financiada casi en su totalidad por el Estado (Egaña, 2001: 130-131). En ese sentido, significó un paso más en dicha consolidación y un claro intento por subsanar el problema ya esbozado por Bello en su crítica hacia el sistema lancasteriano respecto de la idoneidad de los preceptores.

Como hemos señalado, el sistema lancasteriano se basó en el uso de monitores o "niños enseñantes" y con el establecimiento de la Escuela normal se buscó, en primer término, formar una masa crítica de profesores dispuestos y preparados a enseñar la lectoescritura. La fundación de esta institución constituiría, en palabras de Iván Núñez, un intento de "formar maestros idóneos, con reconocida moralidad, provistos de métodos fáciles, claros y uniformes, que debían extender la educación primaria a todas las clases sociales, procurando así ahorro de tiempo" (Núñez, 2010: 135). Manuel Montt diría, un par de años después de su fundación, que "los pocos alumnos que han salido de ella a dirigir escuelas primarias en las provincias contribuirán a uniformar los métodos, a introducir arreglos en los establecimientos" (Montt, 1844: 271).

Sin embargo, a pesar de lo decidido por la Junta de Educación del Instituto Nacional en 1833, la Escuela Normal no "abolió" la vigencia del sistema lancasteriano. En su decreto fundacional estableció, de hecho, que "en esta escuela se enseñarán los ramos siguientes: leer i escribir con perfección, i un conocimiento completo de los métodos de enseñanza mutua i simultánea, dogma i moral relijiosa, gramática i ortografía castellana, jeografía descriptiva; dibujo lineal; nociones jene- 
rales de historia i particulares de la de Chile" (Boletin de los decretos y leyes del gobierno, 1842: 293-294). ${ }^{8}$ Esto implicó, por un lado, reconocer que, en la práctica, el sistema mutuo - que no era otro que el lancasteriano- continuaba funcionando y que se trataba, por tanto, de un sistema válido. Por otro lado, se formalizó algo que apenas se había esbozado en medio del proceso de "abolición" del sistema lancasteriano en el Instituto Nacional una década antes: el uso oficial del sistema simultáneo. En este sentido, el decreto instituyó que un preceptor egresado de la Escuela debía dominar los dos sistemas más importantes. No se habla de un tercer sistema, que en la práctica era el individual, o de un sistema mixto entre ambos.

Que el decreto haya asimilado el sistema mutuo o lancasteriano como uno de estos dos métodos, debe entenderse como el reconocimiento a una práctica que perduró en el tiempo y que refleja la incapacidad del Estado de controlar lo que ocurría en las localidades y lograr una uniformidad como la pretendida en 1821. Como vimos en la sección anterior, para la década de 1830 es posible encontrar testimonios y experiencias que dan cuenta de una realidad en que los actores locales aún no se enteraban de la "abolición" de este sistema en Santiago. Por lo tanto, que el decreto fundacional de la Escuela Normal "reconociera" la vigencia del sistema mutuo, al punto de establecer su enseñanza obligatoria a los futuros maestros, da cuenta de lo difundo que estaba en la práctica. Esto también se debe a que el sistema mutuo o lancasteriano, como el mismo Sarmiento señaló en De la educación popular, era el único que a esas alturas contaba con una definición clara, expresada en decenas de manuales y textos, y, por ende, susceptible de ser estudiado y aplicado. En cambio, el sistema simultáneo, como señala David Hamilton, todavía ofrecía un amplio espacio a interpretaciones, ya que hacia la década de $1830 \mathrm{ni}$ siquiera en Europa existía una definición conceptual precisa acerca de lo que implicaba este sistema. Fue recién a fines de esa década que el sistema simultáneo se identificó como "método pedagógico a través del cual los profesores lograban la atención simultánea de todos sus alumnos" (Hamilton, 1991: 29).

$8 \quad$ El destacado es nuestro. 
Por lo tanto, más que uniformar, el objetivo del reglamento fundacional de la Escuela Normal fue el de acotar los modelos de organización de las escuelas a dos sistemas compatibles con el ideal de la educación masiva: el mutuo o lancasteriano, que en varias localidades era el más conocido, y el simultáneo, que era el que las autoridades deseaban difundir. En su primer informe acerca del estado del establecimiento, fechado el 3 de enero de 1843, Sarmiento aclararía que: "sobre métodos y sistemas de enseñanza no he dado lecciones metódicas por creerlas extemporáneas aún" (Fondo del Ministerio de Justicia, Culto e Instrucción Pública, vol. 12). Optó, en cambio, por adquirir todo el material necesario para que los alumnos de la Escuela pudiesen estudiar los sistemas y métodos por su cuenta. De este modo, el 31 de mayo de 1842 solicitó la adquisición de "la colección de lectura llamada de la enseñanza mutua, $2^{\circ}$ la colección de cuadros del sistema de lectura de Bonifaz, $3^{\circ}$ la clase analítica de Vallejo, $4^{\circ}$ la colección de cuadros de la doctrina de Fleury, $5^{\circ}$ las dos colecciones de aritmética y la de escritura, dispuestas para la enseñanza mutua, hecho con el objeto de hacer una especie de repertorio de todos los métodos de enseñanza conocidos en el país" (Fondo del Ministerio de Justicia, Culto e Instrucción Pública, vol. 12). El propio Manuel Montt, dos años después de la fundación de la Escuela, reforzaría esta visión al señalar que los comisionados del establecimiento "presentan a los preceptores como muy dispuestos a recibir instrucciones i aceptar reformas. No hai pues en estos, apego a prácticas o sistemas; no habrá por consiguiente graves obstáculos para la adopción de un nuevo régimen (...), mas se deja de hacer porque no se sabe que por falta de voluntad" (Montt, 1844: 273).

Con posterioridad, desde las propias provincias se comenzaría a demandar el material necesario para implementar los sistemas de manera adecuada. Desde Ancud, Isidro Salas, el 25 de marzo de 1846, apelaría a falta de maestros y al no envío de " 150 ejemplares del curso de lectura por el método de enseñanza mutua (Fondo de la Intendencia de Chiloé, vol. 27: 26). Por su parte, en 1850, Antonio Cerbelló envió una carta al ministro Salvador Sanfuentes, especificando los útiles que se necesitarían para el establecimiento de la enseñanza mutua en Concepción en su "escuela modelo", que en este caso se refiere a una escuela nocturna. En la carta solicitó el envío de "seis semicírculos de fierro redondo de $31 / 2$ varas de circunferencia (...), 
una colección de cuadros de lectura para la enseñanza mutua, seis pizarras", entre otros (Fondo Ministerio de Justicia, Culto e Instrucción Pública, vol. 33: 49).

En este sentido, la pervivencia del sistema lancasteriano con posterioridad a 1842 debe entenderse como una decisión funcional al objetivo de expandir la instrucción primaria. No obstante, al mismo tiempo, se promovió el sistema simultáneo porque, además ser compatible con dicho anhelo, ofrecía mejores herramientas pedagógicas que el lancasteriano en el desarrollo de los aprendizajes. En este aspecto, Sarmiento mostró una visión pragmática. Si mantuvo y validó el sistema mutuo fue por su capacidad organizativa, especialmente cuando se trataba de un número considerable de alumnos, y por la extensión de su uso. No obstante, su valoración pedagógica del sistema simultáneo era más evidente y no ocultó su preferencia por este en sus escritos. Se conjugaron, entonces, lo ideal con lo real en una primera instancia, para luego transitar a la uniformidad definitiva en torno a un único modelo deseable.

\section{Los visitadores de escuela y la obsolescencia del sistema lancasteriano}

La creación del Ministerio de Instrucción Pública y la fundación de la Escuela Normal de Preceptores y de la Universidad de Chile fueron claros indicios de los esfuerzos del Estado por centralizar la administración del sistema educativo. Aunque esto había surgido como idea en el transcurso de la guerra de Independencia, su concreción se postergó hacia la década de 1840, en la que el Estado docente, definido en la Constitución de 1833, comenzó una gradual consolidación. Sin embargo, el ejercicio de dicho rol dependió de la paulatina construcción de una red de funcionarios a lo largo del territorio, que permitiese al Estado supervisar in situ el funcionamiento del sistema educativo (Soaje y Salas, 2018). De acuerdo con Loreto Egaña, "la búsqueda de la eficiencia, de mejorar la enseñanza, que queda en evidencia en la práctica directa de los visitadores en las escuelas, está en estrecha relación con el ejercicio del poder desde la autoridad central, que impregna completamente esa práctica" (Egaña, 2001: 222). Esto último es relevante, en la medida que fueron estos agentes los que fueron determinando en torno a qué sistema se debía uniformar la enseñanza. 
Si bien el gobierno tenía este objetivo en mente, no se inclinó explícitamente por alguna opción a priori, como lo demuestra este extracto de la Memoria de Manuel Montt: "el gobierno, ejerciendo la dirección general de la instrucción que le corresponde, le dará unidad de acción i la uniformidad en los métodos de enseñanza, que son indispensables para que los esfuerzos comunes produzcan el resultado que se desea" (Monitor de Escuelas Primarias, 15-VIII-1858: 325).

En este objetivo, la creación de los "visitadores de escuela" fue fundamental, dado que sus visitas e informes no solo cumplieron con la misión de observar y supervisar el funcionamiento de las escuelas, sino que aportaron también valiosas opiniones y sugerencias que a la larga pasaron a ser un verdadero insumo para las políticas educacionales del periodo. En ese sentido, no fueron agentes neutrales, sino que solían plantear su propia opinión acerca de lo más conveniente para las escuelas. Como afirma Hillel Davi Soifer, este tipo de agentes fue "fundamental para sistematizar el desarrollo educacional" a través de las presiones que ejercían a sus superiores. El cargo de visitador propiamente tal surgió en 1847, cuando J. D. Bustos fue nombrado visitador para todo el territorio (Egaña, 2001: 223). Paulatinamente, el número de visitadores iría aumentando durante la siguiente década, pero recién con la entrada en vigencia de la Ley de Instrucción Primaria en 1860 , se creó una verdadera red de visitadores con una presencia que abarcaría gran parte del territorio.

En estos informes se puede observar que, a ojos de varios visitadores, se imponía una necesidad urgente de homogeneizar la organización de las escuelas mediante un único sistema. Para algunos autores, se trataría de un esfuerzo homogeneizador en aras de "nacionalizar" a las comunidades que, de manera no explicitada, ofrecerían cierta resistencia a la homogeneización (Ramírez, 2014: 35-52). De ser así, habría que retrotraer ese esfuerzo hacia 1821, cuando, como hemos dicho, se presentó por primera vez un proyecto para homogeneizar a todas las escuelas en torno a un único sistema. En la década de 1850 la realidad era algo distinta, pues, como hemos visto, el reglamento de la Escuela Normal oficializó tanto el sistema mutuo como el simultáneo, y existía una clara preocupación por la calidad del proceso de enseñanza-aprendizaje. Por lo tanto, limitar el rol de los visitadores a un afán nacionalizador es obviar un aspecto básico de su labor, que era 
velar por el desempeño pedagógico de las escuelas y, sobre todo, que los alumnos aprendieran al menos a leer y escribir.

Si bien dichos informes constataban la multiplicidad de sistemas puestos en práctica, al mismo tiempo presentaban una visión crítica de lo que años antes se había denominado el "desorden sistemático". A la luz de ellos, se imponía la necesidad de dotar de una organización y sistematicidad a una escuela que, hacia la década de 1850, todavía mostraba señalas claras de desorganización, la cual, a ojos de visitadores y autoridades, incidía negativamente en el aprendizaje. Asimismo, los informes de los visitadores no solo reflejan preocupación por dicha desorganización, sino que, sobre todo, un diagnóstico claro de que los niños que asistían a las escuelas no estaban aprendiendo debido a la mala aplicación de los sistemas y métodos de enseñanza.

Una primera observación respecto de estos informes es que tampoco ofrecen un corpus homogéneo desde el cual obtener información. No existía un formato único mediante el cual sistematizar y clasificar la información necesaria. Cada visitador elaboró sus informes organizando la información de acuerdo a categorías que estimara pertinentes, por lo que, especialmente en una primera etapa, no todos los informes incorporan información sobre los mismos aspectos. Así, por ejemplo, en 1850, no todos los informes especifican qué sistema de enseñanza utiliza la escuela visitada. En cambio, a partir de 1858 se evidencia una sistematización de la información y una homogeneización de la estructura de los informes mucho más clara y dicha categoría sí comenzaría a incluirse en todos los informes. Como ejemplo, podemos mencionar el informe de Manuel Salas, quien aplicaría un cuestionario a los directores de escuelas de Illapel, con fecha 20 de marzo de 1860, para contar con insumos para su elaboración. Una de las preguntas era: “¿Cuáles son los ramos que se cursan en el establecimiento i que método se sigue en ellos?" La respuesta de todas las escuelas fue que "el método que se sigue es el simultáneo" (Fondo Ministerio de Justicia Culto e Instrucción Pública, vol. 89).

En segundo término, no todos los visitadores hacen explícitas sus preferencias por un determinado sistema, especialmente en una primera etapa. En la mayoría de los informes de 1850-1854 los visitadores expresan una apreciación personal acerca del proceso de enseñanzaaprendizaje al interior de las escuelas, y buscan explicaciones más o 
menos razonables acerca del estado de las mismas. En algunos casos, esta explicación se atribuye a una mala implementación de un sistema, pero no a la implementación de un sistema en sí. En la mayoría de los casos los problemas se atribuyen a dos elementos: la falta de preparación de los preceptores ("nada de disciplina, conocimiento limitadísimo en la preceptora") y la falta de un espacio adecuado y de los recursos mínimos para un funcionamiento aceptable ("el local es tan malo que hace casi imposible todo sistema i ahoga la educación"). Recién a partir de 1858 se observa una política más explícitamente favorable hacia el sistema simultáneo, con el objetivo de transitar hacia la uniformidad respecto de un único sistema en todas las escuelas.

Uno de los informes más completos del periodo es el "Informe sobre el estado actual de la instrucción primaria en el departamento de Santiago", elaborado por Juan Domingo Vico, con fecha 3 de enero de 1850 y organizado en tres cuadernos ${ }^{9}$. En dicho informe Vico analiza la situación de todas las escuelas de Santiago, clasificándolas en conventuales, particulares y fiscales y municipales. De sus observaciones es posible deducir, en primer término, que en Santiago coexistían los tres principales sistemas: individual, simultáneo y mutuo, junto con otros más bien inclasificables. En segundo lugar, las escuelas conventuales y particulares presentan, en general, una situación bastante más "deplorable", "mala" y "lamentable" que las fiscales y municipales. Sobre las conventuales afirmó que "en todas ellas la enseñanza está reducida a un tejido de práctica absurdas i rutineras que nada tienen más en favor que una vetustez inconcebible" (Fondo del Ministerio de Justicia, Culto e Instrucción Pública, vol. 33). Además, tendían a usar con mayor frecuencia el sistema individual que el mutuo o el simultáneo. De ese modo, por ejemplo, de la Escuela de Belén i Matucana y de la Escuela de Clorinda Ferrari afirmó que "no hay más método que el individual". Solo una escuela particular, la de Manuel Barrenechea, utilizaba el sistema simultáneo: "así ha logrado poner en planta el sistema simultáneo, que sin duda es el más acreditado".

En cambio, las fiscales y municipales usaban con mayor frecuencia el sistema simultáneo y el mutuo. Las mejores, a juicio de Vico, eran

9 Archivo Nacional Histórico de Chile, Fondo del Ministerio de Justicia Culto e Instrucción Pública, vol. 33, Santiago, 3 de enero de 1850, fojas 4-40. 
cuatro escuelas, de las cuales tres eran masculinas y una femenina. Todas ellas implementaron el sistema mutuo o lancasteriano de una manera muy cercana a su modelo original. Respecto de las masculinas, la Escuela modelo de La Recoleta, la Escuela de San Diego y la Escuela La Esperanza destacaron por su organización y resultados. De la primera Vico destacó: "el estar mejor provista que ninguna de todos los medios necesarios que favorecen notablemente al sistema de enseñanza, que es el mutuo". Con respeto a la Escuela de la Esperanza especificaría, además, que utilizaba del "método de Lancáster". De ese modo, afirmó sobre el preceptor de la escuela, "caracteriza a este joven una actividad tan adecuada al método de Lancaster, que su influencia se hace sentir de una manera notable hasta en los establecimientos circunvecinos". No obstante, fue para el caso de la Escuela de San Diego que asoció su éxito pedagógico a la posibilidad de implementar un sistema existente, como el lancasteriano o mutuo, con la mayor disponibilidad de elementos necesarios para su funcionamiento:

"puede decirse que el sistema de enseñanza mutua se haya practicado en toda su extensión (...) Los métodos, los monitores, todo aquellos que respecta al mecanismo, a la economía del tiempo, a la moralidad en general i en suma al buen éxito de la enseñanza, forman el principal cuidado del institutor; i hay una infinidad de prácticas que no tienen otro objeto que fomentar en los niños la emulación, el amor propio moderado, la vergüenza i demás medios morales que constituyen la base de todo buen sistema" (Fondo del Ministerio de Justicia, Culto e Instrucción Pública, vol. 33).

En el caso de las escuelas femeninas de La Recoleta y de San Diego, las situaciones eran distintas, ya que, mientras la primera también utilizó el sistema mutuo, a lo que atribuyó una "disciplina bastante regular", la segunda implementó el individual. Una explicación de por qué el sistema mutuo tendía a ser más utilizado en las escuelas fiscales y municipales era su mayor número de alumnos. De hecho, en su informe con fecha 24 de abril de 1860, es decir, casi diez años después del informe de Vico, el visitador Víctor Gutiérrez diría algo similar respecto de las escuelas fiscales y municipales del departamento de Osorno. Todas ellas implementaban el sistema mutuo, con todos los elementos necesarios para su funcionamiento y siguiendo casi al pie de la letra el criterio de Lancaster para formar cada curso o clase al interior de la 
escuela: "en las escuelas fiscales i municipales se sigue el sistema de enseñanza mutua, valiéndose el preceptor de monitores, distribuidos los niños en clases o secciones conforme al tiempo en que entraron a la escuela i consiguientemente al grado de su saber". Esto difería de la escuela particular fundada por colonos alemanes, "en la que se sigue el sistema simultáneo, por el cual los niños no están subdivididos en clases, sino que todos van a la par". Para Gutiérrez esto era inaplicable, debido a que "los niños chilenos con sus continuas fallas se quedan atrás en el aprendizaje, mientras otros van adelante i así la escuela es un sinnúmero de divisiones i subdivisiones de clases de diferente grado de adelanto" (Fondo del Ministerio de Justicia, Culto e Instrucción Pública, vol. 91).

Paulatinamente, los informes irán explicitando cada vez más la necesidad de uniformar los sistemas y métodos de enseñanza, además de constatar su uso y las razones del mayor o menor progreso en las escuelas. Así, por ejemplo, en La Serena, el 10 de marzo de 1854 se comisionó a Pablo Silva, alumno de la normal, como visitador, "con el fin de uniformar la enseñanza i hacer a los preceptores las indicaciones i prevenciones que hagan más acertada su dirección i provechosos sus servicios" (Fondo del Ministerio de Justicia, Culto e Instrucción Pública, vol. 59). Este ejemplo comienza a evidenciar también la estrecha vinculación entre la Escuela Normal y los visitadores, ya que en su mayoría comenzarían a ser egresados de dicha institución. En este caso se nominó a un preceptor de dicha escuela, con el exclusivo objeto de uniformar los sistemas de enseñanza de las escuelas de La Serena, sin mencionar una preferencia por alguno de los sistemas vigentes.

A partir de 1858 los informes serán cada vez más explícitos en este objetivo y comenzarán también a publicarse no solo informes acerca del estado de las escuelas, sino que varias propuestas de reforma por parte de los mismos visitadores, con el objetivo de mejorar la enseñanza. Varias propuestas se centrarán en la uniformidad de los sistemas y métodos, con clara preferencia por alguno, aunque por diferentes motivos. De este modo, para el visitador de escuelas de Concepción, al referirse a las escuelas fiscales de hombres, uniformar los métodos aparecerá como algo deseable, no así la iniciativa de cada preceptor: "la uniformidad en los métodos de enseñanza era desconocido (...) La 
distribución del tiempo era viciosa, porque cada preceptor la determinaba como mejor le parecía o estimaba conveniente" (Monitor de Escuelas Primarias, 15-III-1858: 181). En el caso del visitador de Talca, además de manifestar su concordancia con la necesidad de uniformar la enseñanza, propuso que esto no solo implicara aplicar un método o sistema ya conocido, sino que se tomara en cuenta también la experiencia de los visitadores y su potencial contribución a generar incluso un nuevo sistema educativo a partir de sus observaciones: "la creación de visitadores para todas las provincias de la República parece que ha tenido por objeto uniformar los métodos de enseñanza (...) con la práctica en la inspección de las escuelas, con las observaciones que cada cual hace en su trabajo, puede ser que se altere algun tanto esa uniformidad tan necesaria para el progreso, a que algunos más privilegiados que los demás mejoren los métodos ya existentes o quizás inventen alguno nuevo que reportase un gran provecho" (Monitor de Escuelas Primarias, 15-VI-1858: 271).

Sin embargo, hacia la década de 1860, los informes muestran que la mayor parte de los visitadores comenzó a manifestar una clara preferencia por dos sistemas: el simultáneo y el mixto simultaneo-mutuo. Esta preferencia nació como consecuencia de la observación y de la constatación de que, en la práctica, eran los sistemas que mejor se amoldaban al contexto de cada provincia y a la realidad material de las escuelas. Un factor relevante era que, como había señalado Sarmiento, el sistema mutuo se amoldaba mejor a escuelas numerosas, de más de cien alumnos, pero la realidad de la mayoría de las escuelas en Chile era de una cifra inferior a esa. Por lo tanto, y siguiendo también el criterio de que el sistema simultáneo se adaptaba mejor a escuelas de tamaño intermedio con menos de cien alumnos, los visitadores comenzaron a proponer este sistema, o su variación mixta, como la solución ideal para reformar la enseñanza. Para ello, el recurso de un reglamento propuesto por los mismos visitadores fue el mecanismo predilecto. Este fue el argumento tras la decisión de uniformar en torno al sistema simultáneo a todas las escuelas fiscales i municipales de la provincia de Coquimbo mediante un reglamento: "Art. $6^{\circ}$ : se adoptará en todas las escuelas la enseñanza simultánea, por convenir este método a todo establecimiento de 20 a 100 alumnos" (Monitor de Escuelas Primarias, 15-VI-1858: 283). 
No obstante, en varias provincias se optó por la variación mixta de este sistema. En abril de 1858 el visitador de Chiloé señaló que "el deseo de uniformar la enseñanza por cuanto se puede en toda la provincia se trató de llenarlo con un reglamento" (Monitor de Escuelas Primarias, 15-IV-1858: 200). Aunque no explicita un sistema, el informe presentado dos años después para la misma provincia dio clara cuenta de que en todos los departamentos de Chiloé (Castro, Ancud, Carelmapu, Quimchao) se implementaba el sistema simultáneo o el simultáneo mutuo (Monitor de Escuelas Primarias, 15-VI-1860: 262-279). Esta constatación llevó posteriormente a regular esto mediante un reglamento, que fue común tanto para Arauco como para Chiloé: "art. 28. el sistema de enseñanza que por hora se practicará en las escuelas de Chiloé i Arauco es el conocido bajo el nombre de simultáneo mutuo" (Monitor de Escuelas Primarias, 15-XI-1860: 325). En el informe del visitador de escuelas de Talca se señala que en dicha provincia "la educación es simultánea mixta, es su método" (Monitor de Escuelas Primarias, 15-XI-1859: 35). En Concepción, el visitador también se pronunció explícitamente con respecto al método de enseñanza que debía seguir funcionando en la provincia: "de los tres sistemas de reconocida utilidad, el simultáneo, el mutuo i el misto, he preferido para prescribirlo [el misto] en todas las escuelas de esta provincia (...) El misto pone en práctica por sí solo todo lo útil de los dos primeros" (Monitor de Escuelas Primarias, 15-III-1861: 171). Francisco Bascuñán, visitador en Santiago, afirmaría el 24 de abril 1861 que "el sistema adoptado en las escuelas gratuitas es el mixto compuesto del mutuo i simultáneo. En las particulares se sigue el simultáneo participando en algunas de muy pequeñas prescripciones del mutuo (Fondo del Ministerio de Justicia, Culto e Instrucción Pública, vol. 98).

Lo anterior cobra relevancia también en la medida en que la Ley de Instrucción Primaria de 1860 modificó la aproximación hacia los sistemas educativos. En lugar de prescribir, como lo hacía el Reglamento de la Escuela Normal de Preceptores de 1842, los sistemas o métodos específicos que un preceptor debía conocer, la ley estipuló en su artículo $9^{\circ}$ que en las escuelas normales "se enseñará (...) pedagojía teórica y práctica", sin hacer referencia a ningún sistema. Posteriormente, en 1863, esta idea sería incorporada en el nuevo Reglamento de la Escuela Normal de Preceptores, cuyo artículo 45, referido a la enseñanza impartida por la institución, replicaba la misma fórmula. Esto significa que ninguna nor- 
mativa decretó el fin del sistema lancasteriano o mutuo, sino que, por una parte, se dejaron de prescribir como obligatorios sistemas o métodos específicos y, por otra, fue la activa labor de los visitadores lo que impulsó la uniformidad en torno al sistema simultáneo.

Este proceso muestra que, si bien el sistema lancasteriano o mutuo de enseñanza perduró más allá de su "abolición", en 1833, decretada por la Junta de Educación del Instituto Nacional, paulatinamente fue perdiendo vigencia en favor del sistema simultáneo y, a lo sumo, fue un componente de una versión híbrida de este. En general, se puede observar que las críticas pedagógicas, si bien fueron relevantes, no mellaron su vigencia en algunas localidades. Sin embargo, lo que se implementaba en ellas, a ojos de los visitadores, distaba mucho de ser una versión fidedigna, sobre todo por la escaza cantidad de alumnos de las escuelas, que no se condecía con la naturaleza del sistema lancasteriano, creado como un mecanismo de educación masiva, con capacidad para enseñar a cientos de alumnos a la vez.

\section{Conclusión}

En este artículo hemos intentado demostrar que el sistema mutuo o lancasteriano de enseñanza tuvo una vigencia en Chile mayor a la que tradicionalmente le asigna la historiografía. Si bien se adoptó como parte sustantiva de un proyecto educacional ligado a un nuevo paradigma político republicano y constituyó una suerte de enlace con el "mundo moderno y civilizado", su vigencia se vio enfrentada a la crítica pedagógica surgida en diferentes momentos con posterioridad a la Independencia. Esto no impidió que, al igual que en otros países hispanoamericanos, el sistema lancasteriano se mantuviera como un elemento articulador en la construcción del sistema educativo chileno, incluso hacia la segunda mitad del siglo XIX. En parte, esto se debió a que su difusión lo convirtió en un sistema ampliamente conocido y a que era funcional al objetivo de masificar la educación primaria en un contexto en que el Estado todavía no instalaba la capacidad para fomentar y supervisar dicha masificación.

El análisis de este proceso de continuidad nos permite comprender también los mecanismos mediante los cuales se mantuvieron ciertas prácticas pedagógicas, las cuales también experimentaron procesos de adaptación en los entornos locales. Esto se debió, en 
gran medida, a la "libre interpretación" de los preceptores, que mantuvieron su implementación con anterioridad al surgimiento de una institucionalidad que ejerciera control sobre las prácticas. Esto abrió el suficiente espacio para implementar el sistema en la medida que se conociera y que las condiciones lo permitieran, y para que algunos de sus elementos se combinaran con elementos del sistema simultáneo. En ese sentido, más que hablar de "un" sistema mixto, durante este periodo se implementó un mosaico de sistemas educativos, lo que fue de alguna manera a contracorriente de la política estatal, la cual apuntó a uniformar gradualmente los sistemas de enseñanza. De esta manera, a partir de la fundación de la Escuela Normal de Preceptores y la consecuente labor de los visitadores de escuela en su rol de agentes estatales, se observa un claro intento del Estado por controlar la organización interna de las escuelas en aras de mejorar la calidad de la enseñanza, pero también de uniformar y homogeneizar en torno a un único sistema deseable. La activa labor de los visitadores fue fundamental para determinar en terreno qué sistema era implementado adecuadamente de acuerdo con las condiciones ideales de funcionamiento y fue en ese proceso de observación, crítica y retroalimentación que se llegó a la conclusión de que el sistema que se adaptaba de mejor manera a las condiciones locales era el sistema simultáneo. En este sentido, fue precisamente el fortalecimiento de la institucionalidad estatal para ejercer el control y la supervisión lo que explica el gradual abandono de este sistema.

\section{Referencias bibliográficas}

Fuentes primarias

a) Fondos documentales

Archivo Histórico del Liceo Gregorio Cordovez, Biblioteca Regional Gabriela Mistral, La Serena, vol. 2.

Archivo Nacional Histórico de Chile, Fondo Ministerio de Justicia Culto e Instrucción Pública, vols. 4, 12, 17, 33, 59, 89, 91, 98.

Archivo Nacional Histórico de Chile, Fondo Ministerio del Interior, vol. 45.

Archivo Nacional Histórico de Chile, Fondo Intendencia de Concepción, vols. 107, 116, 160, 274, 394. 
Archivo Nacional Histórico de Chile, Fondo Intendencia de Coquimbo, vol. 299.

Archivo Nacional Histórico de Chile, Fondo Intendencia de Chiloé, vol. 27.

b) Fuentes impresas

Henríquez, C. (1822). Bosquejo compendioso del sistema de enseñanza mutua. Colección Manuscritos de Camilo Henríquez. Santiago: Biblioteca Nacional de Chile.

Lancaster, J. (1803). Improvements in education: as it respects the industrious classes of the community: containing, among other important particulars, an account of the institution for the education of one thousand poor children, Borough Road, Southwark, and of the new system of education on which it is conducted. Londres: Darton and Harvey.

Lancaster, J. (1810). British System of Education: Being a Complete Epitome of the Improvements and Inventions Practised at the Royal Free-Schools. Londres: Royal Free School, Borough Road.

Montt, M. (1841). Memoria que el Ministro del despacho de Justicia, Culto e Instrucción Pública presenta al Congreso Nacional. Santiago: Imprenta y Litografía del Estado.

Montt, M. (1844). Memoria que el Ministro del despacho de Justicia, Culto e Instrucción Pública presenta al Congreso Nacional. Santiago: Imprenta y Litografía del Estado.

Prieto, J. (1833). “Discurso del Presidente de la República a las Cámaras legislativas", en Sesiones de los cuerpos legislativos de la República de Chile, vol. XXI (1831-1833). Sesión ordinaria del 1 de junio de 1833, pp. 383-384.

Prieto, J. (1834). “Discurso del Presidente de la República a las Cámaras legislativas", en Sesiones de los cuerpos legislativos de la República de Chile, vol. XXIII (1834-1836). Sesión del Congreso Nacional, 5 de junio de 1824, pp. 23-29.

Prieto, J. (1836). “Discurso del Presidente de la República en la apertura de las cámaras legislativas de 1836", vol. XXV (1836-1839). Sesión del Congreso Nacional, 1 de junio de 1836, pp. 4-6.

Sarmiento, D. (2009). De la educación popular (Primera edición de 1849). Santiago: Biblioteca Fundamentos de la Construcción de Chile. 
Sarmiento, D. (1899). Obras de D. F. Sarmiento. Vol. 28: Ideas pedagógicas. Buenos Aires: Imprenta y Litografía Mariano Moreno.

Thomson, J. 1827. Letters on the Moral and Religious State of South America, written during a residence of nearly seven years in Buenos Aires, Chile, Peru and Colombia. Londres: James Nisbet.

\section{c) Publicaciones periódicas}

Boletín de los decretos y leyes del gobierno, vol. 10, N², I-1842.

Monitor de Escuelas Primarias, Santiago, 1858-1861.

\section{Fuentes secundarias}

a) Artículos y capítulos de libros

Baeza, A. (2018). "Presentación: Bosquejo compendioso de enseñanza mutual de Camilo Henríquez", en Cuadernos Chilenos de Historia de la Educación, Nº, pp. 180-190.

Baeza, A. (2015). "Educational Reform, Political Change, and Penury: Primary Schooling and the Monitorial System of Education in Chile, approx. 1810-1833", en M. Caruso (ed.). Classroom Struggle. Organizing Elementary School Teaching in the 19th Century. Frankfurt: Peter Lang, pp. 67-90.

Baeza, A. (2017). "One Local Dimension of a Global Project: The Introduction of the Monitorial System of Education in PostIndependent Chile, 18211833", en Bulletin of Latin American Research, vol. 36, №3, pp. 340-353.

Browning. W. (1921). "Joseph Lancaster, James Thomson, and the Lancasterian System of Mutual Instruction, with Special Reference to Hispanic America", en Hispanic American Historical Review, vol. 4, pp. 49-98.

Caruso, M. (2015). "Classroom Struggle. Organizing Elementary Teaching in the 19th Century", en M. Caruso (ed.), Classroom Struggle. Organizing Elementary Teaching in the 19th Century. Frankfurt: Peter Lang, pp. 9-40.

Caruso, M. y E. Roldan (2005). "Pluralizing Meanings: The Monitorial System of Education in Latin America in the Early Nineteenth-Century", en Paedagogica Historica, vol. 41, $\mathrm{N}^{\circ} 6$, pp. 645-654.

Hamilton, D. (1991), "De la instrucción simultánea y el nacimiento de la clase en el aula", en Revista de Educación, №296, pp. 23-42. 
Hassard, J, y M. Rowlison (2002). “Researching Foucault's Research: Organization and Control in Joseph Lancaster's Monitorial Schools", en Organization, vol. 9, №4, pp. 615-639.

Iglesias-Rogers G. (2017). “José Joaquín de Mora in Chile: From NeoEurope to the 'Beocia Americana”, en Bulletin of Latin American Research, vol. 36, N³, pp. 327-339.

Lamperiere, A. (2017). “¿Excepcionalidad chilena? La formación del Estado entre revolución e institucionalización", en I. Jaksic y F. Rengifo (Editores). Historia política de Chile. Tomo II: Estado y Sociedad. Santiago: Fondo de Cultura Económica, Universidad Adolfo Ibáñez, pp. 23-54.

Landhal, J. (2019). "Learning to Listen and Look: The shift From the Monitorial System of Education to Teacher-led Lessons", en The Senses and Society, vol. 14, N², pp. 194-206.

Mayorga, R. (2017). “Una red educativa. Cuatro escuelas, millones de ciudadanos: Educación, estado republicano y sociedad civil en Chile (1813-2011)", en I. Jaksic, y F. Rengifo (Editores). Historia política de Chile. Tomo II: Estado y Sociedad. Santiago: Fondo de Cultura Económica, Universidad Adolfo Ibáñez, pp. 333-364.

Newland, C. (1991). "La educación elemental en Hispanoamérica: Desde la independencia hasta la centralización de los sistemas educativos nacionales", en Hispanic American Historical Review, vol. 71, №2, pp. 335-364.

Núñez, I. (2010). “Escuelas normales: una historia larga y sorprendente. Chile (1842-1973), en Pensamiento educativo, vols. 46-47, pp. 133-150.

Ossa, J. L. (2007). "La actividad política de Francisco Antonio pinto: 1823-1828. Notas para una revisión biográfica", en Historia, vol. 1, N40, pp. 91-128.

Ponce de León, M. (2010). "La llegada de la escuela y la llegada a la escuela. La extensión de la educación primaria en Chile, 18401907", en Historia, vol. 43, N², pp. 449-486.

Racine, K. (2008). “The British and Foreign Bible Society's Interest in Spanish America, 1805-1830", en M. Brown (ed.) Informal Empire in Latin America. Culture, Commerce and Capital. Blackwell Publishing: Oxford, pp. 78-98.

Racine. K. (2010). “This England and This Now: British Cultural and Intellectual Influence in the Spanish American Independence 
Era”, en Hispanic American Historical Review, vol. 90, N³, pp. 423-454.

Racine. K. (2020). “Monitors and Moralists: The Lancasterian System of Mutual Education and the Vision of a New Moral Order in Spanish America, 1818-1831", en History of Education, vol. 49, N², pp. 143-159.

Ramírez, E. (2014). “Dominación. La escuela, los visitadores y los niños. Chile, 1840-1860", en Revista de Historia, vol. 1, №21, pp. 35.52 .

Roldán, E. (2011). “Internacionalización pedagógica y comunicación en perspectiva histórica: La introducción del método de enseñanza mutua en Hispanoamérica independiente," en M. Caruso y H. Tenorth (ed.), Internacionalización. Políticas educativas y reflexión pedagógica en un medio global. Buenos Aires: Gránica, pp. 297-344.

Roldán, E. (2005). “ ¿Order in the Classroom’: The Spanish American Appropriation of the Monitorial System of Education", en Paedagogica Historica, vol. 4, N6, pp. 655-675.

Sedra, P. (2010). "Exposure to the Eyes of God: Monitorial Schools and Evangelicals in Early Nineteenth-century England", en Paedagogica Historica, vol. 47, N³, pp. 263-281.

Silveira, A. (2013). “Educating a City's Children: British Immigrants and Primary Education in Buenos Aires (1820-1880)", en The Americas, vol. 70, $\mathrm{N}^{\circ} 1$, pp. 33-62.

Soaje, R. y M. Salas (2018). “José Bernardo Suárez: un agente de la educación popular y su visión sobre la instrucción primaria en Chile (1867)", en Revista de Historia y Geografía, №39, pp. 49-72.

Soifer, H. (2009). “The Sources of Infrastructural Power: Evidence from Nineteenth-Century Chilean Education", en Latin American Research Review, vol. 44, N², pp. 158-180.

Tanck de Estrada, D. (1973). "Las escuelas lancasterianas en la ciudad de México: 1822-1842", en Historia mexicana, vol. 22, N4, pp. 494-513.

Toro, P. (2018). "Ideas políticas educacionales en Chile, c.1810-c.1980", en I. Jaksic y S. Gazmuri (Editores), Historia politica de Chile. Tomo IV: Pensamiento político. Santiago: Fondo de Cultura Económica, Universidad Adolfo Ibáñez, pp. 103-132. 
Tschurenev, J. (2008). "Diffusing Useful Knowledge: The Monitorial System of Education in Madras, London and Bengal, 17891840", en Paedagogica Historica, vol. 44, N³, pp. 245-264.

\section{b) Libros}

Aedo, R. (2000) La educación privada en Chile. Un estudio históricoanalítico desde el periodo colonial hasta 1990. Santiago: RIL Editores.

Amunátegui, D. (1895). El sistema de Lancaster en Chile y otros países sudamericanos. Santiago: Imprenta Cervantes.

Amunátegui, D. (1889). Los primeros años del Instituto Nacional (18131835). Santiago: Imprenta Cervantes.

Collier, S. (1967). Ideas and Politics of Chilean Independence. 18081813. Cambridge: Cambridge University Press.

Egaña, M. (2001). La educación primaria popular en Chile en el siglo XIX: Una práctica de política estatal. Santiago: Ediciones DIBAM.

Espinoza, A. (2013). Education and the State in Modern Peru. Primary Schooling in Lima, 1821-1921. Nueva York: Palgrave-MacMillan.

Jakisc, I. (2001). Andrés Bello: la pasión por el orden. Santiago: Editorial Universitaria.

Gutiérrez, C. 2011. Educación, ciencia y artes en Chile, 1797-1843. Santiago: RIL Editores.

Kaestle, C. (1973). Joseph Lancaster and the Monitorial School Movement. A documentary History. Londres y Nueva York: Teachers College Press.

López, E. (2014). El proceso de construcción estatal en Chile. Hacienda pública y burocracia (1817-1860). Santiago: Centro de Investigaciones Diego Barros Arana.

Pita, R. (2017). Patria, Educación y Progreso: El impulso a las escuelas y colegios públicos en la naciente República de Colombia, 18191828. Bogotá: Academia Colombiana de Historia.

Roldán, E. (2003). The British Book Trade and Spanish American Independence. Education and Knowledge Transmission in Transcontinental Perspective. Aldershot: Ashgate.

Serrano, S. (2016). Universidad y Nación. Chile en el silgo XIX. $2^{\circ}$ edición. Santiago: Editorial Universitaria. 
Serrano, S., M. Ponce de León y F. Rengifo (2012). Aprender a leer y escribir (1810-1880). Vol. 1. Historia de la Educación en Chile (1810-2010). Santiago: Editorial Taurus.

Twells, A. (2008). The Civilising Mission and the English Middle Class, 1792-1850. The 'Heathen' at Home and Overseas. Londres: Palgrave.

Vaughan, E. (1987). Joseph Lancaster in Caracas 1824-1827: And His Relations with the Liberator Simon Bolivar: With Some Accounts of Lancasterian Schools in Spanish America in the Nineteenth Century and Some Notes on the Efforts of the British and Foreign Bible Society to Distribute the Scriptures in Spanish in the Same Territory. Caracas: Ministerio de Educación.

Weinberg, G. (1984). Modelos educativos en la historia de América Latina. Buenos Aires: UNESCO, CEPAL, PNUD, 1984. 